Conclusions Significant numbers of children have a low or critically decreased body fat mass. This problem is very vital and has to be evaluated as a highly dangerous risk factor for health and prospective life quality of the children. It is recommended to use FMI when analysing changes in body mass. Hyperdiagnosics of adiposity occurs when BMI is used.

\section{P2-428 H1N1 INCIDENCE AND RATE OF COMPLICATIONS IN PREGNANT WOMEN DURING THE 2009/10 WINTER PANDEMIC}

doi:10.1136/jech.2011.142976l.58

${ }^{1} \mathrm{~S}$ V Katikireddi, ${ }^{*}{ }^{1} \mathrm{D}$ G Mackenzie, ${ }^{2} \mathrm{P}$ Warner, ${ }^{2} \mathrm{~L}$ Williams, ${ }^{1} \mathrm{~K}$ Templeton, ${ }^{3} \mathrm{~W}$ Carman, ${ }^{1} \mathrm{~K}$ Smith, ${ }^{3} \mathrm{~W}$ Adamson, ${ }^{1} \mathrm{P}$ Dewart, ${ }^{1} \mathrm{~K}$ Dundas, ${ }^{4} \mathrm{~F}$ Denison. ${ }^{1} \mathrm{NHS}$ Lothian, Edinburgh, Scotland, UK; ${ }^{2}$ Centre for Population Health Sciences, University of Edinburgh, Edinburgh, Scotland, UK; ${ }^{3}$ West of Scotland Specialist Virology Centre, Glasgow, Scotland, UK; ${ }^{4}$ Simpson Centre for Reproductive Health, University of Edinburgh, Edinburgh, Scotland, UK

Introduction Case series suggest pregnant women are at increased risk of contracting H1N1 and experiencing complications. Published studies to date have investigated symptomatic patients or ascertained serology cross-sectionally. Such studies do not allow accurate quantification of incidence and neglect mild disease when estimating complication rates. We investigated $\mathrm{H} 1 \mathrm{~N} 1$ incidence and rate of complications in unvaccinated women in Scotland during the winter 2009/10 pandemic.

Method We recruited 417 unvaccinated pregnant women who attended hospitals in NHS Lothian in Dec 2009-April 2010. Participants completed a research nurse-administered questionnaire, had venous blood taken and clinical outcomes were extracted from hospital records. Booking blood samples (collected routinely at 10-14 weeks gestation) were retrieved for each participant to allow testing of paired blood samples using the microneutralisation assay. Evidence of infection during pregnancy was defined as a 10-fold increased in H1N1 antibody titre between booking and delivery.

Results Seroconversion between booking and delivery occurred in $10.5 \%$ (95\% CI $7.1 \%$ to $13.9 \%$ ) with 19 of 32 unaware of acquiring infection. Self-reporting flu symptoms and asthma (but no other chronic conditions) were statistically significant predictors of infection. No significant differences were found in rates of maternal or neonatal hospital admission, critical care admission, birth weight or adverse events between those infected and uninfected.

Conclusion In Scotland where estimated coverage of H1N1 vaccination in pregnancy was $47.6 \%$, relatively few unvaccinated pregnant women experienced H1N1 infection with many infected experiencing minimal symptoms. No increased risk of adverse events was detected but we have low power for this analysis.

\section{P2-429 PREVALENCE OF INFLUENZA A (H1N1) SEROPOSITIVITY IN UNVACCINATED HEALTHCARE WORKERS IN SCOTLAND AT THE HEIGHT OF THE GLOBAL PANDEMIC}

doi:10.1136/jech.2011.142976l.59

${ }^{1} \mathrm{~K}$ Smith, ${ }^{2} \mathrm{~L}$ Williams, ${ }^{3} \mathrm{~W}$ Adamson, ${ }^{1} \mathrm{~S} V$ Katikireddi, ${ }^{1} \mathrm{P}$ Dewart, ${ }^{1} \mathrm{E}$ Fletcher, ${ }^{3} \mathrm{~W}$ Carman, ${ }^{1} \mathrm{~K}$ Templeton, ${ }^{4} \mathrm{~F}$ Denison, ${ }^{2} \mathrm{P}$ Warner, ${ }^{1} \mathrm{D} \mathrm{G}$ Mackenzie. ${ }^{1} \mathrm{NHS}$ Lothian, Edinburgh, Scotland, UK; ${ }^{2}$ Centre for Population Health Sciences, University of Edinburgh, Edinburgh, Scotland, UK; ${ }^{3}$ West of Scotland Specialist Virology Centre, Glasgow, Scotland, UK; ${ }^{4}$ Simpson Centre for Reproductive Health, University of Edinburgh, Edinburgh, Scotland, UK

Introduction Susceptibility of healthcare workers (HCW) to influenza is relevant in terms of sickness absence, productivity and onward transmission of infection from carer to patient, a particular issue with a novel influenza strain. The aim of this study was to determine the seroprevalence of antibodies against the virus in unvaccinated healthcare workers in Lothian, south east Scotland during October- November 2009 (after the first wave of infection, but before the expected increase in cases over the winter period).

Methods Unvaccinated employees $(n=493)$ were recruited within days of the start date of the HCW vaccination programme and prior to most being vaccinated. Participants had a serum sample taken and completed a short questionnaire recording sex, age, occupation, and self-reported history of flu-like symptoms or illness since the start of the pandemic period. Serology specimens were analysed in the West of Scotland Specialist Virology Centre in Glasgow using microneutralisation assays at a dilution of 1:40.

Results The prevalence of seropositivity in HCWs mid-pandemic was $10.3 \%$ (95\% CI 7.7 to $13.0 \%$ ), which was higher than prepandemic HCW seropositivity rate by $3.7 \%$ (95\% CI of increase $0.3 \%$ to $7.3 \%, p=0.048)$. Seropositivity rates for frontline and nonfrontline HCWs were similar.

Conclusion To our knowledge, this is the first study in the United Kingdom to quantify the level of seropositivity to influenza A (H1N1) in unvaccinated HCWs pre- and mid-pandemic. Only $10.3 \%$ of HCWs were seropositive for influenza $\mathrm{A}(\mathrm{H} 1 \mathrm{~N} 1)$ mid-pandemic, so the great majority were still susceptible to infection at the introduction of the vaccination programme.

\section{P2-430 WHO/TDR NEW DENGUE GUIDELINE WORKING BETTER FOR PATIENT CARE}

doi:10.1136/jech.2011.142976l.60

M Khursheed, K Ejaz, J Razzak, U R Khan. ${ }^{*}$ Aga Khan University Hospital, Karachi, Sindh, Pakistan

Introduction Globally Dengue has threatened to infect 3 billion people. TDR/WHO version launched the latest version of Dengue guidelines recently. In this cross sectional study, we compared clinical diagnosis of patients admitting to the hospital with WHO 1997 and TDR/WHO 2009 guidelines. To also explored strengths and weakness of the two classifications.

Methodology Dengue cases admitted from January 2005 to December 2007 at Aga Khan University Hospital, Pakistan were reviewed. Data were recoded using Dengue grading according to the WHO 1997 and TDR/WHO 2009 guidelines. Correlation among the three sets of disease classifications were tested statistically.

Results TDR/WHO 2009 in comparison to the clinical classification showed that out of 612, 24 (4\%) patients did not fulfil the new classification. TDR labelled 124 (20\%) patients as having Severe Disease, out of which 118 (95\%) were labelled as DF, $3(2.5 \%)$ as DHF and 3 $(2.5 \%)$ as DSS by the physician. $\left(\chi^{2} 18.7, p\right.$ value 0.005$)$ (Likelihood Ratio 17.9, p value 0.006) Comparing the new guidelines with the old, TDR labels $124(20 \%)$ cases as Severe Disease in comparison to the 24 $(4 \%)$ by WHO. ( $\chi^{2} 89.8$, p value 0.0001 ) (Likelihood Ratio 92.1, pvalue 0.0001$)$ The value of 0.24 of Cramer's $V$ signifies that there is little association between the two classifications.

Conclusion TDR/WHO 2009 dengue guideline is a better version of WHO 1997 guideline as it is able to identify the critical patients early in the disease course. However, this might lead to overestimation of disease severity which can be a restraint for developing nation's resources.

\section{P2-431 MARITAL STATUS AND RISK OF HIV INFECTION IN INFORMAL URBAN SETTLEMENTS OF NAIROBI, KENYA RESULTS FROM A CROSS-SECTIONAL SURVEY}

doi:10.1136/jech.2011.142976l.61

${ }^{1} \mathrm{~J}$ Kimani, ${ }^{*} \mathrm{R}$ Ettarh, ${ }^{2} \mathrm{~A}$ Ziraba, ${ }^{3} \mathrm{~N}$ Yatich. ${ }^{1}$ The African Population and Health Research Center, Nairobi, Kenya; ${ }^{2}$ Faculty of Epidemiology and Population Health, 